\title{
A Methodology for Optimal MAC Protocol Generation: Case Study of a Synchronous MAC Channel
}

\author{
Jian Zhen, Forrest Brewer, and Volkan Rodoplu \\ Department of Electrical and Computer Engineering \\ University of California, Santa Barbara \\ Santa Barbara, CA 93106
}

\begin{abstract}
We describe a methodology that incorporates the impact of control information transfer into MAC protocol optimization. We apply this methodology to the problem of a synchronous broadcast MAC channel, to generate the optimal protocol when the objective function is the average network throughput per slot. We describe a recursive procedure for the symbolic generation of the optimization program for any choice of the objective function. We demonstrate that this methodology produces the Random Access Channel and protocols with control information as special cases in the regimes where they are optimal. We examine the scaling of optimal throughput and of computational complexity as a function of the number of nodes and the control lifetime.
\end{abstract}

Index Terms-medium access control, network protocol, design automation

\section{INTRODUCTION}

${ }^{1}$ The automated design of networking protocols is a new approach to protocol design, with substantial potential benefits to reduce the protocol design cycle, labor costs, and design errors while delivering high-performance protocols in a wide range of application-specific contexts [1]. Just as the advent of the silicon compiler brought unprecedented benefits to the design of integrated circuits in the 1980's, design automation of protocols is poised to bring similar benefits to the design of networks. Medium Access Control (MAC) is one of the critical layers in wireless networks, with direct impact on protocol performance (throughput, energy consumption, or delay), and one that can benefit immensely from protocol automation. Our aim in this paper is to demonstrate, through a case study, a methodology by which the design of wireless MAC protocols can be automated.

In the past, the main approach to reconfigurable protocol design has been to "choose" a certain protocol or family of protocols for the application at hand. Further, via analysis [3][5] or simulation/emulation studies [4], one can describe the protocol family that performs the best under different scenarios. For example, for wireless MAC protocols, if the control overhead is large compared to data, a random access channel (RAC) protocol would have better performance, and if the control overhead is small compared to data, a CSMA/CA protocol would have better performance due to its ability to

\footnotetext{
${ }^{1}$ This work was supported in part by the National Science Foundation Grant \# 0917052.
}

reserve future data slots with high probability. In contrast, in protocol generation, since the impact of control information is incorporated into the optimization program, the "optimal" protocol is generated, taking the RAC and RTS-CTS protocols as special cases in the larger protocol space.

There have been only a few works in the past that have attempted to generate protocols automatically while extremizing an objective function. First, there is the work on the automatic generation of security protocols [6][7]. The second body of related work is layering via optimization decomposition [8], which does not incorporate control into optimization, and produces optimal results for the regime where data is much longer than control. However, for wireless networks, in particular for MAC protocols for wireless sensor networks, the control information exchanges and data take up comparable resources. In contrast, the framework in this paper incorporates the impact of control into optimization.

References [1] and [2] proposed a framework that incorporates the impact of control information exchanges between the nodes into the optimization of a single-neighborhood MAC protocol, for the performance metrics of throughput and energy respectively. Even though the asynchronous nature of transmission with propagation delays was addressed, these formulations used deterministic optimization variables to model the transmission schedules of the nodes. Deterministic optimization variables cannot handle the mixture of random access and advertised transmission schedules, and the optimal solutions, which will be identical for identical nodes, will produce continuous collisions when followed by each node. These references solved this problem by purposely breaking the symmetry between the nodes and creating a pre-determined chain of leaders and followers that determine the order in which the control will be transmitted. However, such presumed leader election, if resolved, should practically obviate the MAC problem in the first place. Hence, a different representation of node knowledge states is required, that successfully captures the mixture of random access and deterministic transmission modes under the same optimization framework. In this paper, we derive that representation and the corresponding optimization framework.

We establish the following methodology: (1) The impact of successful transmission of control information is represented 
by the "knowledge state" of a node, that encodes what control information the node has sent into the channel, and what control information it has received. Note that because these are wireless links, a node can never be sure that the control it has sent has been perfectly received. (2) The knowledge states of a node form a Markov chain, and the probability that the node takes action $a$ upon knowledge state $s$ is an optimization variable. It is key to note that these variables are defined only with respect to the local information available at that node, which is encoded in its knowledge state. (3) The Cartesian product of the Markov chains of all the nodes is the global knowledge state representation of the network. The optimizer uses this global state representation, even though no individual node has access to the global state representation. (4) Based on the symmetries of the network (such as the case of identical nodes), the global state representation is mapped to an equivalent representation, called the "reduced global state representation". This is a significant step in reducing the total number of variables of the program, as well as the computation of the symbolic expression of the objective function. (5) An objective function is given exogenously (e.g. average network throughput) as a metric defined on each arc or state of the Markov chain. It is essential to note that no symbolic expression of the objective function is given a priori (since its number of terms scales with the size of the Markov chain). (6) The objective function is symbolically computed recursively by a breadth-first search down the reduced global state representation, and the optimization program is symbolically generated. (7) The generated optimization program is solved via state-of-the-art non-linear solvers.

The rest of the paper is organized as follows: In Section II, we carry out Steps (1)-(4) of the above methodology for the case study of a synchronous broadcast MAC with any number of nodes $N$. In Section III, we carry out Steps (5)-(7) of the above methodology, continuing the same case study. In Section IV, we display and discuss the results generated by this methodology, and validate them via mathematical analysis in their asymptotic regime. In Section V, we present our conclusions and discuss directions for future work.

\section{REPRESENTATION OF KNOWLEDGE StATES AND} TRANSITIONS

We focus on the following case study to demonstrate the above methodology: $N$ identical nodes, with infinitely long data in their MAC buffers, aim to access a single MAC neighborhood (i.e. all nodes can hear each other). We assume that nodes have slot synchronization, but that they do not have any knowledge of each other's future transmissions, unless advertised through a control packet. The set of actions of each node is identical and is given by $\mathcal{A}=\{d, n, \hat{d}, \hat{n}\}$, where $d$ stands for sending data in that slot, $n$ for listening, $\hat{d}$ for sending a control packet into the channel that announces to the other nodes that this node will send data in the following $W$ slots, and $\hat{n}$ for sending a control packet into the channel that announces to the other nodes that this node will listen to the channel for the next $W$ slots. Note that we use broadcast

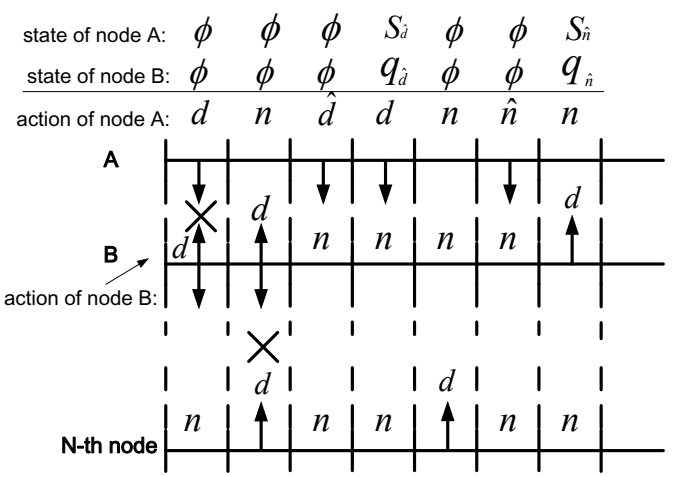

Fig. 1. An illustration of the node actions and states, for $\mathrm{W}=1$.

transmission for both data and control packets; hence, if a control packet is sent successfully, it is heard by all of the $N-1$ nodes. In addition, note that $W$ is a fixed number for simplicity, and can be advertised within a control packet. We call $W$ the "control lifetime", namely the number of slots in the immediate future over which the control packet has any effect. In our case, $W$ is the same for both $\hat{d}$ and $\hat{n}$ control packets, but can be made to be different for a more general optimization program. We take our optimization metric to be the average throughput, namely the long-run average of successful data transmissions per slot.

Fig. 1 shows an example of single-neighborhood MAC operation of such a network, with $W=1$. In the first slot, both nodes $A$ and $B$ transmit data, and their transmissions collide, wasting that slot for all of the nodes in the network. In the second slot, $A$ is silent, but $B$ collides with the $n$th node. In the third slot, $A$ sends $\hat{d}$, which advertises that it will send data $d$ in the next $W$ slots $(W=1$ here). As a result, $A$ 's knowledge state in the next slot goes from $\phi$ (the null knowledge state) to $S_{\hat{d}}$, which indicates that node $A$ has just sent $\hat{d}$ in the previous slot. In this example, all of the either nodes were quiet in the third slot, and $A$ 's control packet successfully got through to all of the nodes, and since control packets are transmitted in broadcast mode, each of these nodes switches from $\phi$ to $q_{\hat{d}}$ which indicates that it has just received a control information packet $\hat{d}$. Due to the broadcast mode, there is no need to mark from whom this control packet was received. Note that node $A$ 's switching to knowledge state $S_{\hat{d}}$ depends only on $A$ 's sending $\hat{d}$ in the previous slot, and cannot depend on successful reception of this $\hat{d}$ (which happens to be the case in this example), of which $A$ can have no knowledge due to the wireless nature of the channel. No acknowledgments are modeled; however, they can be added to the model under this methodology at the cost of increasing the complexity of the protocol space.

The global knowledge state of the network at any time, is the Cartesian product of the Markov chains of these $N$ nodes. In Fig. 2, we have drawn the Markov chain for the global knowledge state of the network for $N=2$ and $W=1$. The doubles in the state bubbles correspond to the 
pairs of the knowledge states of the individual nodes. In this diagram, there are 4 states besides the $\phi$ knowledge state: These states correspond to the cases where one of the nodes has successfully sent control (either $\hat{d}$ or $\hat{n}$ ) to the other node (indicated by $S_{\hat{d}}$ or $S_{\hat{n}}$ respectively), and the other node has received this control packet, and hence transitioned in its own state space to a $q_{\hat{d}}$ or $q_{\hat{n}}$ state.

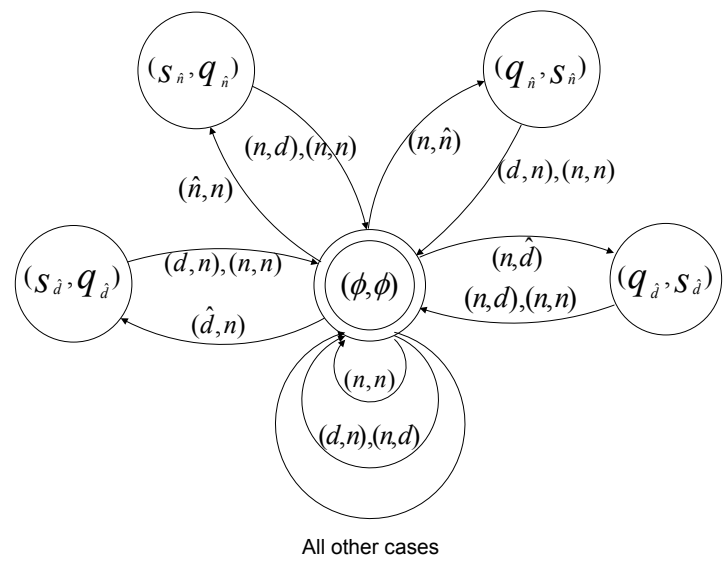

Fig. 2. Global State Space for $\mathrm{N}=2, \mathrm{~W}=1$

For the general case of $N$ nodes, both the knowledge states of the nodes and the global state given by their Cartesian product are well-defined, however, difficult to draw. Based on this general description, an important state space reduction occurs (Step 5 of the Methodology) when we note that due to the broadcast nature of the control packet transfer, the state of a node is uniquely given by one of the following 5 states, $\mathcal{S}=\left\{\phi, s_{\hat{d}}, s_{\hat{n}}, q_{\hat{d}}, q_{\hat{n}}^{m}\right\}$, where $\mathcal{S}$ denotes the state space of an individual node. The first three states correspond to the null knowledge state, the state where a node has just sent $\hat{d}$, and the state where the node has just sent $\hat{n}$. The fourth state indicates that the node has received a $\hat{d}$ somewhere in the last $W$ slots. This is the correct generalization of the above example for $W=1$ to the case of the general $W$. Since $\hat{d}$ promises that this node will follow this control packet with $W$ slots of data, a node can have received at most one $\hat{d}$ in the last $W$ slots. In contrast to this, a node may have received multiple $(m) \hat{n}$ 's in the last $W$ slots; hence, the fifth state in the list is a set of states parameterized by $m$ where $q_{\hat{n}}^{m}$ indicates that this node has received $m \hat{n}$ 's in the last $W$ slots, where $0 \leq m \leq \min (N-1, W)$. (We let $q_{\hat{n}}^{0}=\phi$.)

In general, we denote the "action set" of a node, namely the set of actions that a node can take in each slot, by $\mathcal{A}$, and the state space of a node by $\mathcal{S}$. We let $\theta_{a \mid s}$, where $a \in \mathcal{A}$ and $s \in \mathcal{S}$, denote the conditional probability that the node takes action $a$ in state $s$. These $\theta_{a \mid s}$ 's are the optimization variables of our program.

Based on the above reduced representation of the global state space, the transition rules from one global state to another are simply given as follows: If a $\hat{d}$ control packet has been transmitted successfully some time in the last $W$ slots, which we represent by $I_{\hat{d}}$, then the node that has advertised this will be sending data $d$ in the next slot. Even though all of the other nodes have the flexibility to take any action they want in the next slot, if they transmit anything in the next slot, they will collide with the $d$, and if all of them listen, then they will receive that $d$ successfully. Either of these cases cannot cause a knowledge state transition in that slot for any of these nodes. As a result, the global state remains in $I_{\hat{d}}$ with probability 1. For all other cases, the probability of transition from one global state to the next, for any action $a \in \mathcal{A}=\{n, d, \hat{n}, \hat{d}\}$, is given by:

$$
\left(\begin{array}{c}
N-m \\
1
\end{array}\right) \cdot \theta_{a \mid q_{\hat{n}}^{m}} \cdot \theta_{n \mid q_{\hat{n}}^{m}}^{N-m-1} \cdot \theta_{n \mid s_{\hat{n}}}^{m},
$$

where $m$ is the number of $\hat{n}$ control packets that have been sent by all nodes in the last $W$ slots, and $\theta_{n \mid s_{\hat{n}}}=1$.

\section{Symbolic Generation OF The Optimization PROGRAM}

We may impose any metric of our choice on this Markov state space. In this paper, we shall maximize the average network throughput per slot. The network scores a throughput of 1 unit whenever only a single node sends data $(d)$ in a slot, and all of the other nodes are quiet. Note that $\hat{d}$ does not score any throughput in that slot. (It is easy to generalize this to a model where the control information consumes only a fraction $\alpha$, e.g. the header of a packet, by assigning a throughput of $1-\alpha$ to every arc where $\hat{d}$ is transmitted.)

The average throughput (reward) per slot on a Markov chain is calculated as follows: Define a "cycle" of a recurrent Markov chain as the pair of events from the time that the chain starts in a recurrent state to the time that the chain returns to the same state (for the first time). Let $T$ denote the duration of this cycle. (Note that $T$ is a random variable.) Let $F$ denote the reward that the chain collects (on its arcs) in that cycle. That, independent of the choice of the recurrent state, the average reward per slot is computed as $E[F] / E[T]$. Hence, our first task is to automatically generate the expression for this objective function.

We use a breadth-first recursive algorithm, shown in Alg.1, to accumulate and generate the symbolic expressions for $E[F]$ and $E[T]$, using the null knowledge state as the initial state of the cycle. Because the cumulative probability of a path diminishes with the number of hops in the state space, we use a maximum search depth $N_{\text {sd }}$. In this way, we trade off optimization accuracy with computation complexity.

Fig. 3 provides a picture of the state space through which the recursive algorithm branches out in its calculation. The key point is that many of the states, even though they reside in the state space, are not reachable states. Since the transitions to those states are never computed in the breadth-first search, this approach obviates any a priori pruning of unreachable states from the state space. The algorithm starts from $S 0$ (the null knowledge state), and calls the recursive function AccumulateMetrics(). In the base case, the algorithm checks the stop condition (line 11 of the Algorithm): (1) it has 


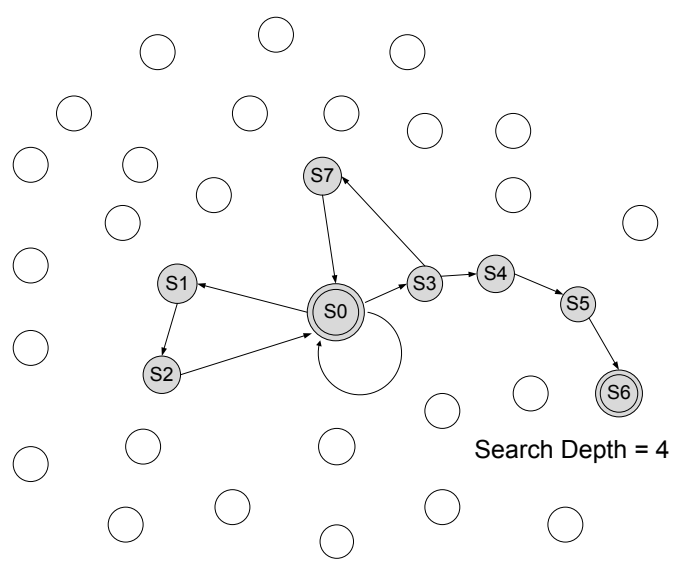

Fig. 3. Recursive accumulation of the metrics in the AccumulateMetrics() function

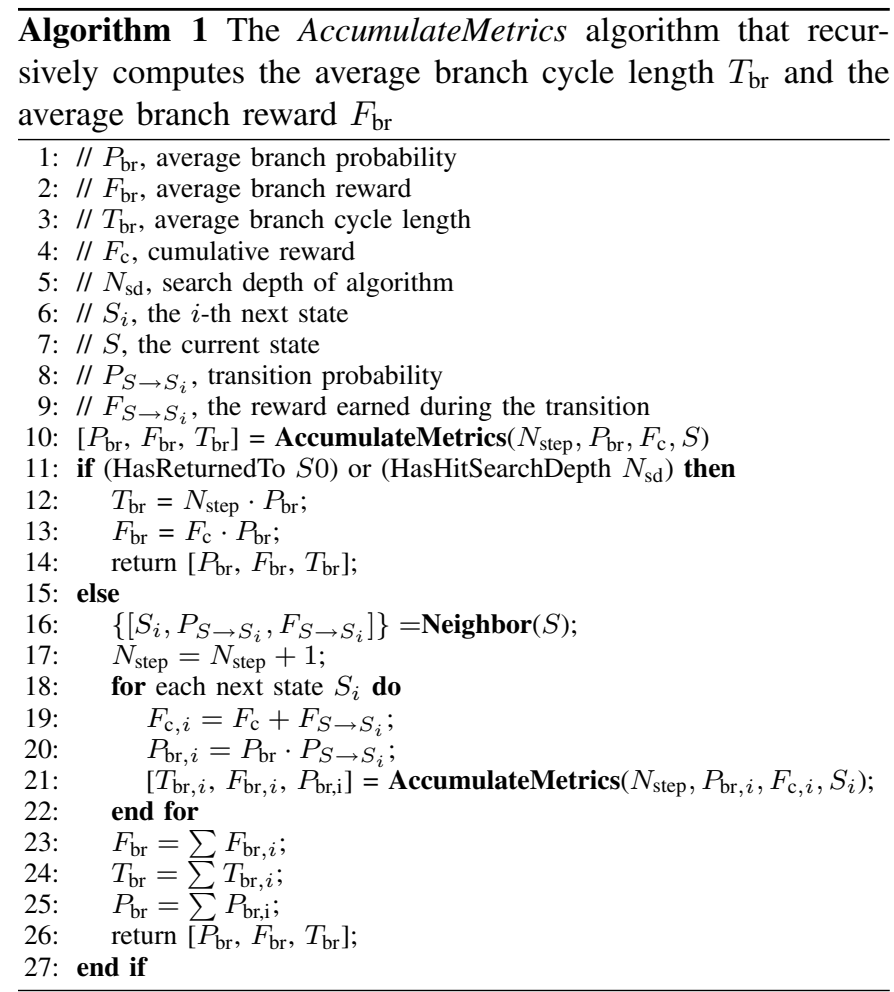

returned to the initial null knowledge state, or (2) It has arrived at the search depth $N_{\mathrm{sd}}$. If the stop condition is satisfied, the algorithm returns the average branch probability, average branch cycle length, and the average branch reward. Otherwise, the algorithm calls the function Neighbor() to calculate the set of valid states to which it can transition, with associated transition probabilities and rewards, then accumulates the rewards and multiplies the probabilities for each next state; after that, AccumulateMetrics() function is called recursively for each next state. The total reward and total cycle length are calculated on lines 23, 24, and 25.

We have written the automatic expression generation in MATLAB, using its support for symbolic expressions. An ex- ample objective function generated this way is given below, for $N=10$, and $W=2$, for network throughput maximization (The objective function is $E[F] / E[T]$ ):

$$
\begin{aligned}
\mathrm{E}[F]= & \theta_{n \mid \phi}^{9}\left(10 \theta_{d \mid \phi}+20 \theta_{\hat{n} \mid \phi}+180 \theta_{\hat{n} \mid \phi} \theta_{n \mid q_{\hat{n}}}^{8} \theta_{d \mid q_{\hat{n}}}\right. \\
& +90 \theta_{\hat{n} \mid \phi} \theta_{n \mid q_{\hat{\hat{n}}}}^{8} \theta_{\hat{d} \mid q_{\hat{n}}}-810 \theta_{\hat{n} \mid \phi} \theta_{n \mid q_{\hat{n}}}^{16} \theta_{d \mid q_{\hat{n}}} \theta_{\hat{n} \mid q_{\hat{n}}} \\
& -810 \theta_{\hat{n} \mid \phi} \theta_{n \mid q_{\hat{n}}}^{16} \theta_{d \mid q_{\hat{n}}} \theta_{\hat{d} \mid q_{\hat{n}}} \\
& \left.+720 \theta_{\hat{n} \mid \phi} \theta_{n \mid q_{\hat{n}}}^{8} \theta_{\hat{n} \mid q_{\hat{n}}} \theta_{n \mid q_{\hat{n}}^{2}}^{7} \theta_{\hat{d} \mid q_{\hat{n}}^{2}}\right) \\
\mathrm{E}[T]= & 20 \theta_{n \mid \phi}^{9} \theta_{\hat{n} \mid \phi}+20 \theta_{n \mid \phi}^{9} \theta_{\hat{d} \mid \phi}+1
\end{aligned}
$$

subject to the following constraints: $\theta_{d \mid s_{\hat{d}}}=1, \theta_{n \mid s_{\hat{n}}}=1$, $0 \leq \theta_{a \mid s} \leq 1$, and $\forall a \in \mathcal{A}, \sum_{a} \theta_{a \mid s}=1$. We use MATLAB's fmincon function, which uses sequential quadratic programming, to solve the resulting objective function. This function is non-linear since it is a rational function of two polynomials, and all of the constraints that specify that the probabilities $\theta_{a \mid s}$ that emanate from state $s$ add up to 1 , are linear. Hence, the result is a non-linear program, that exhibits local maxima. As a result, the choice of the starting point for the sequential quadratic program is crucial. One approach that has very good empirical performance is to use a Monte Carlo simulation, treating the non-linear optimization program as a complex system. When the starting points are chosen randomly within the feasible set, and the maximum is retained, the procedure converges to the global optimal value with high probability; however, the convergence time may be long. A better procedure, which we use in the next section, is to start from the optimal solution of the network with one fewer number of nodes. This procedure is shown to have fast convergence, and is validated, in the large $W$ regime, to converge to the optimal value obtained by asymptotic analysis.

\section{RESUlTS AND Discussion}

Our goal in this section is to analyze the scaling of both performance of the optimal solutions produced for throughput maximization as well as the computational complexity of those solutions, as a function of the number of nodes $N$ and the control lifetime $W$.

Fig.4(a) displays the optimal transition probabilities computed via our methodology as a function of $N$, for $W=5$, for the null knowledge state. We see that the probability that a node listens in a slot is $\theta_{n \mid \phi}=1-1 / N$; that is, the optimal probability that a node is silent in this model where control information can be sent, is the same as it would be for the pure Random Access Channel. Further, we see that the probability that control information $\hat{d}$ is sent when the node is in the null knowledge state is $1 / N$, and no data $d$ is ever sent without preceding it with a control packet $\hat{d}$, for $W=5$; that is, $\theta_{d \mid \phi}=0$. What is important to notice here is that this optimal decision was generated by our methodology, and did not need to be hand-designed.

Fig.4(b) displays the optimal transition probabilities from the null knowledge state $\phi$, for $N=5$, as a function of $W$. We see that the probability that a node is silent is $1-1 / 5=0.8$ for all $W$, and that a "phase transition" occurs from $W=1$ 


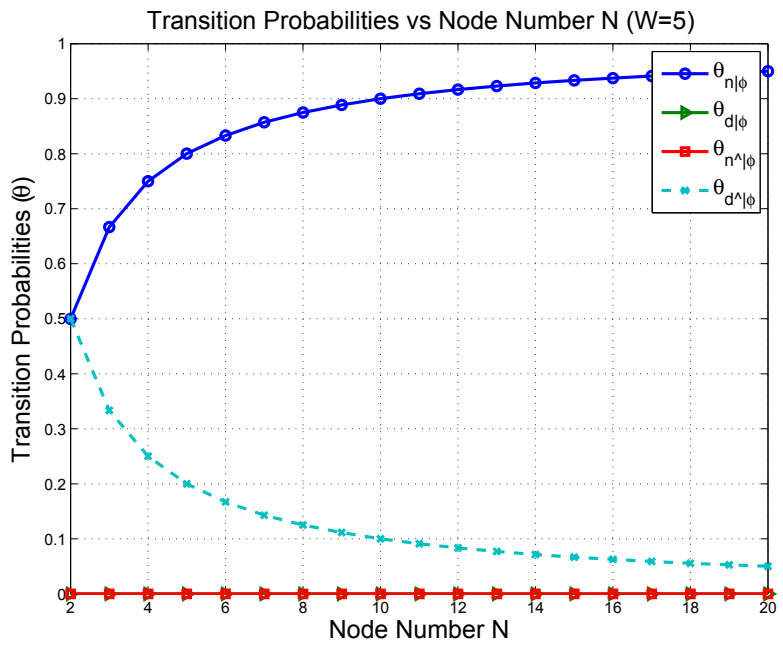

(a)

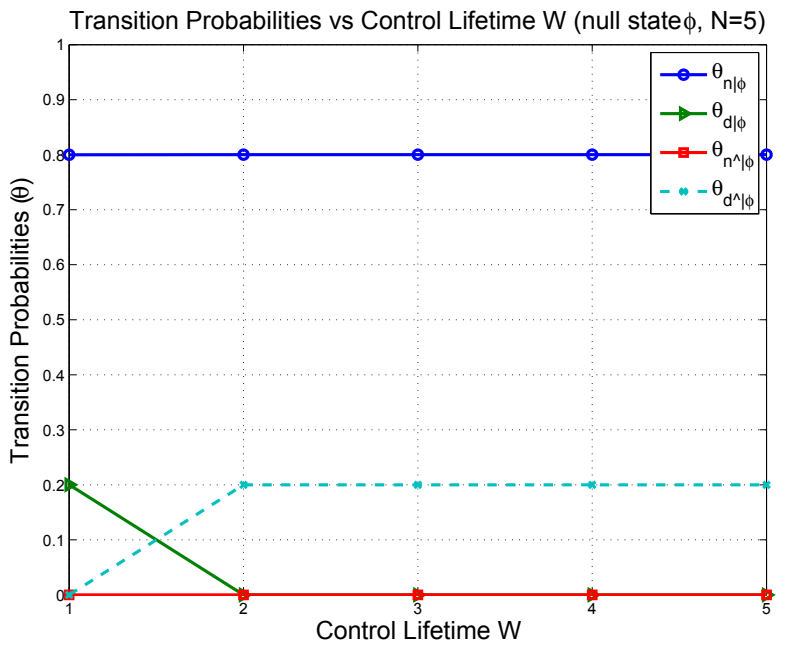

(b)

Fig. 4. (a) Optimal transition probabilities versus the number of nodes $N$, for $W=5$ (b) Optimal transition probabilities versus control lifetime $W$, for $N=5$

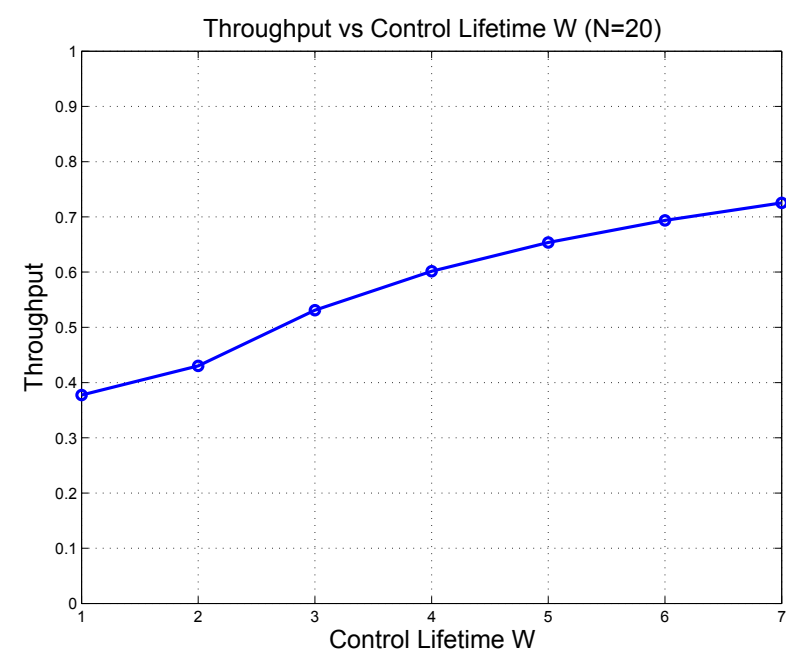

Fig. 5. Network throughput versus the control lifetime $W$, for $N=20$

to $W=2$, where the optimal decision switches from a pure Random Access Channel, to one that always precedes data with $\hat{d}$. Because control information consumes 1 slot, it is not worth sending $\hat{d}$, if the length of the data that will follow it is not long enough. It is important to note that this decision was automatically generated by our methodology, and if the optimization metric changes, which results in a new switching point, the optimal solution for that case, which is different, can quickly be generated. Neither a carefully hand-designed protocol nor any mathematical analysis is needed to derive the switching point. The main effort goes into the general formulation and the symbolic generation of the optimization program, and the result is quickly re-configurable for different metrics.

Fig.5 displays the optimal average throughput as a function of $W$. We see that throughput increases monotonically as a function of $W$, and the inflection point that occurs at $W=2$ corresponds to the phase transition that occurs when the optimal solution switches from a pure Random Access Channel solution to one that always utilizes control. The monotonic increase as a function of $W$ is due to the fact that the successful transmission of $\hat{d}$, when it occurs, allows the other nodes to know to keep quiet, which continues to increase the average throughput as $W$ increases, albeit with diminishing returns. We shall analytically show below, that $\lim _{W \rightarrow \infty} \bar{F} \equiv E[F] / E[T]=1$; that is, this graph converges to 1 ; however, the rate at which it converges $\partial \bar{F} / \partial W$ goes as $1 / W$, which is very slow. Practically, we would decide on a $W$, e.g. 8, beyond which the returns are not worth the increase in complexity.

Fig. 6(a) displays the optimal average throughput as a function of the number of nodes $N$, parameterized by $W$. We see that the optimal average throughput decreases with $N$, albeit flattening out quickly, and for each $N$, monotonically increases with $W$. We can validate these results for the large $W$ regime by the following analysis: Let $K$ denote the number of slots until the first successful transmission of $\hat{d}$ in the network. As $W$ becomes large, the node who sent the $\hat{d}$ will send data for a very long time $(W)$; hence, the average throughput will be approximately $\bar{F} \approx \frac{W}{W+\mathrm{E}[K]}$. Since the probability of that $\hat{d}$ is transmitted successfully is $p_{N} \equiv(1-1 / N)^{N-1}$, and $K$ has a geometric distribution with this success probability, $\bar{F} \approx \frac{W}{W+\frac{1}{(1-1 / N)^{N-1}}}$ for large $W$. Hence, $\lim _{N \rightarrow \infty} \mathrm{E}[\bar{F}] \approx \frac{W}{W+e}$, which concurs perfectly with all of the obtained results that appear for $N=20$ in Fig. 6(a). From the same expression, it follows that $\lim _{W \rightarrow \infty} \bar{F} \approx 1$, for every $N$, which shows the asymptotic limit in Fig. 5 is 1 . Calculating the convergence rate $\partial \bar{F} / \partial \bar{W}$ as a function of $p_{N}$ gives a maximum of $1 / 4 \mathrm{~W}$, which shows the rate at which it approaches the asymptote of 1 . It is important to note that this 


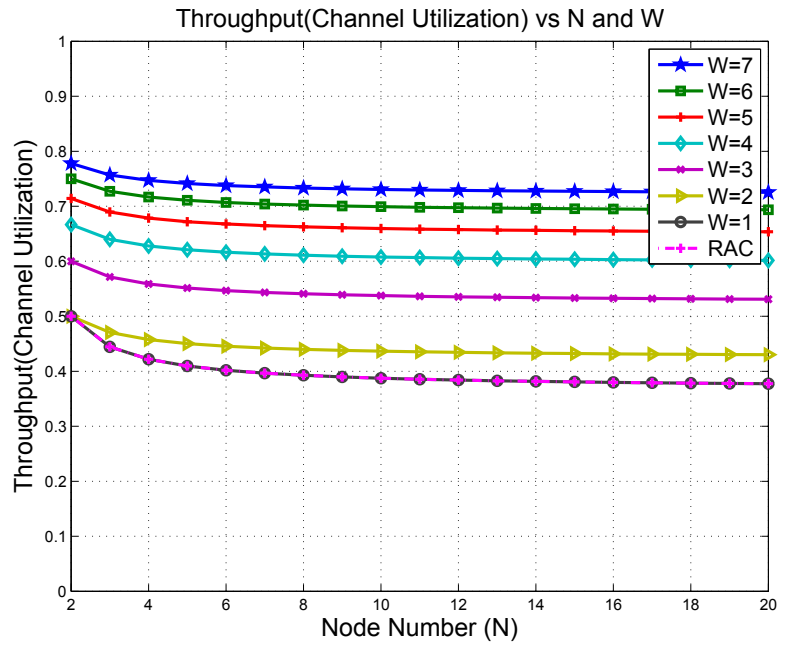

(a)

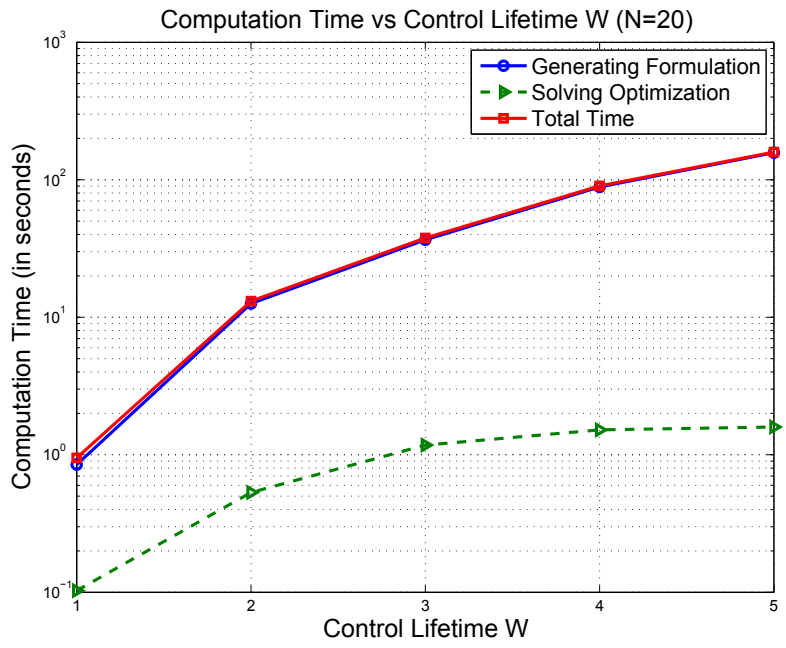

(b)

Fig. 6. (a) Network throughput as a function of the number of nodes, parameterized by the control lifetime $W$. (b) Computation time versus the control lifetime $W$, for $N=20$

mathematical analysis is not necessary for our methodology to work, and only serves as a cross-validation for our results.

We have collected the measurements of the computational complexity by measuring the execution time of the entire procedure in MATLAB, when the process was run on a Dell Studio 540 Mini-Tower, with Intel Core 2 Quad Processor Q9550 (2.83GHz, 1333MHz FSB) and 12MB cache.

Fig.6(b) displays the plots for both the time needed for the symbolic generation of the optimization program, and the process of solving the optimization program, using the tictoc feature of MATLAB, when the program was run on a Dell Studio 540, in the absence of any other active computationally intensive processes. The plot shows that the generation of the symbolic expression of the optimization program dominates the total computation time. (This will be reduced by performing the symbolic generation in a native language such as $\mathrm{C}$, rather than in an interpreter such as MATLAB.) For this case study, because this is a broadcast channel, the number of states in the reduced global state space remains the same as $N$ grows; hence, the complexity of symbolic generation is roughly constant in $N$. If control information were unicast, computational complexity would grow with $N$.

\section{CONClusions And Future Work}

The methodology that we have developed in this paper can be applied to any choice of an objective function defined on the global state space, and the same symbolic generator can be invoked to produce the program. For example, if the objective is to minimize the average energy consumption subject to a minimum throughput constraint for each node, then adding 1 line of code in the constraints, and changing a single line in the recursive function, quickly produces the new optimal protocol. This is in sharp contrast with hand-designed protocols that would design differently for each metric.
Our future work directions are: (1) Expanding the protocol space to (a) asynchronous MAC protocols, (b) with Acknowledgments, and (c) overlapping MAC neighborhoods, while keeping the complexity of symbolic generation low. It is key to note that the complexity incurred is mostly is generating the compact formulation than in solving it. (2) In this larger protocol space, we expect that protocols such as CSMA/CA will turn out to be special cases in the feasible set of protocols.

\section{REFERENCES}

[1] V. Rodoplu, and A. Aminzadeh Gohari, "Challenges: Automated Design of Networking Protocols," in Proc. of ACM International Conference on Mobile Computing and Networking (MobiCom'08), Sept. 2008.

[2] V. Rodoplu, A. Aminzadeh Gohari, and W. Tang, "Towards Automated Design of MAC Protocols for Underwater Wireless Networks," in Proc. of the 3rd ACM International Workshop on Underwater Networks (WUWNET'08), Sept. 2008.

[3] Sinem Coleri Ergen, Piergiuseppe Di Marco, Carlo Fischione, "MAC Protocol Engine for Sensor Networks," in Proc. of the IEEE Global Communications Conference (Globecom' 09), Nov. 2009.

[4] Joseph Polastre, Jason Hill and David Culler, "Versatile Low Power Media Access for Wireless Sensor Networks" in Proc. ACM SenSys, New York, 2004, pp. 95107

[5] Kurtis B. Kredo II and Prasant Mohapatra, "A hybrid medium access control protocol for underwater wireless networks," In Proceedings of the ACM International Conference on Underwater Networks (WUWNet), pages 33-40, September 2008

[6] A. Perrig and D. Song, "A First Step towards the Automatic Generation of Security Protocols," in Symposium on Network and Distributed Systems Security (NDSS), Feb. 2000.

[7] D. Song, A. Perrig, and D. Phan, "AGVI - Automatic Generation, Verification, and Implementation of security protocols," in Computer Aided Verification: Proc. 13th Intl. Conf. (CAV 2001), G. Berry, H. Comon, and A. Finkel, Eds., Lecture Notes in Computer Science, Vol. 2102, Berlin, Germany: Springer-Verlag, 2001, pp. 241-245.

[8] M. Chiang, S.H.Low, A.R. Calderbank, and J. C. Doyle, "Layering as optimization decomposition: A mathematical theory of network architecture," in Proceedings of IEEE, vol.95, no.1, pp. 255-312, 2007. 